\title{
Hypergravity hinders axonal development of motor neurons in Caenorhabditis elegans
}

\author{
Saraswathi Subbammal Kalichamy ${ }^{1}$ ， Tong Young Lee ${ }^{1}$ ， Kyoung-hye Yoon ${ }^{1}$ ， Jin Il Lee ${ }^{\text {Corresp. } 1}$ \\ ${ }^{1}$ Division Of Biological Science and Technology, Yonsei University, Wonju, South Korea \\ Corresponding Author: Jin II Lee \\ Email address: jinillee@yonsei.ac.kr
}

As space flight becomes more accessible in the future, humans will be exposed to gravity conditions other than our $1 \mathrm{G}$ environment on Earth. Our bodies and physiology, however, are adapted for life at $1 \mathrm{G}$ gravity. Altering gravity can have profound effects on the body, particularly the development of muscles, but the reasons and biology behind gravity's effect are not fully known. We asked whether increasing gravity had effects on the development of motor neurons that innervate and control muscle, a relatively unexplored area of gravity biology. Using the nematode model organism Caenorhabditis elegans, we examined changes in response to hypergravity in the development of the 19 GABAergic DD/ND motor neurons that innervate body muscle. We found that a high gravity force above 10G significantly increases the number of animals with defects in the development of axonal projections from the DD/VD neurons. We showed that a critical period of hypergravity exposure during the embryonic/early larval stage was sufficient to induce defects. While characterizing the nature of the axonal defects, we found that in normal $1 G$ gravity conditions, DD/VD axonal defects occasionally occurred, with the majority of defects occurring on the dorsal side of the animal and in the mid-body region, and a significantly higher rate of error in the 13 VD axons than the 6 DD axons. Hypergravity exposure increased the rate of DD/VD axonal defects, but did not change the distribution or the characteristics of the defects. Our study demonstrates that altering gravity can impact motor neuron development. 
1

2 Title: Hypergravity hinders axonal development of motor neurons in Caenorhabditis elegans

3

4 Authors: Saraswathi Subbammal Kalichamy, Tong Young Lee, Kyoung-hye Yoon, Jin Il Lee

5

6

7 Affiliations: Division of Biological Science and Technology, College of Science and

8 Technology, Yonsei University, Wonju, 220-710, South Korea.

9

10 Correspondence should be addressed to:

11 Jin I. Lee (jinillee@yonsei.ac.kr)

12

13

14 


\section{ABSTRACT}

As space flight becomes more accessible in the future, humans will be exposed to gravity conditions other than our $1 \mathrm{G}$ environment on Earth. Our bodies and physiology, however, are adapted for life at $1 \mathrm{G}$ gravity. Altering gravity can have profound effects on the body, particularly the development of muscles, but the reasons and biology behind gravity's effect are not fully known. We asked whether increasing gravity had effects on the development of motor neurons that innervate and control muscle, a relatively unexplored area of gravity biology. Using the nematode model organism Caenorhabditis elegans, we examined changes in response to hypergravity in the development of the 19 GABAergic DD/VD motor neurons that innervate body muscle. We found that a high gravity force above $10 \mathrm{G}$ significantly increases the number of animals with defects in the development of axonal projections from the DD/VD neurons. We showed that a critical period of hypergravity exposure during the embryonic/early larval stage was sufficient to induce defects. While characterizing the nature of the axonal defects, we found that in normal $1 \mathrm{G}$ gravity conditions, DD/VD axonal defects occasionally occurred, with the majority of defects occurring on the dorsal side of the animal and in the mid-body region, and a significantly higher rate of error in the $13 \mathrm{VD}$ axons than the 6 DD axons. Hypergravity exposure increased the rate of $\mathrm{DD} / \mathrm{VD}$ axonal defects, but did not change the distribution or the characteristics of the defects. Our study demonstrates that altering gravity can impact motor neuron development. 


\section{INTRODUCTION}

Human anatomy and physiology is well adapted for survival and fitness on the planet earth and its stable gravitational conditions. Altering gravity can have profound impacts on the human body. This is especially relevant with the possibility of long-term space travel and habitation and the associated changes in gravity in different space environments. Although some of the effects of decreased gravity on the human body are identified, such as muscle atrophy (Vandenburgh et al. 1999), the genetic, molecular and cellular basis of gravity-induced changes is not well known.

One of the cellular processes that may be constrained by gravity conditions is neuronal development. Previous work showed that microgravity induced a decrease in synaptic density in the hindlimb area of the motor cortex in the brains of rats that were aboard a space mission for 16 days (DeFelipe et al. 2002). Alterations in gravity could also affect the development of neuronal projections. In hypergravity conditions, $150 \mathrm{G}$ force induced neuron-like cell differentiation and development of longer neurites in cultured PC12 cells (Genchi et al. 2015). Moreover, low gravity during spaceflight can induce alterations in dendritic arbor development in medial spinal motor neurons in rats that innervate and control muscle (Inglis et al. 2000). Although altering gravity can affect motor neuron development, the genetic and molecular mechanism of gravity's effect on neuron development is unknown.

4 The nematode C. elegans is one of the premier metazoan genetic model organisms, and studies with the worm have led to seminal discoveries including RNA interference, microRNAs, and genes controlling programmed cell death (Fire et al. 1998; Hengartner \& Horvitz 1994; Lee et al. 1993). Moreover, because worms are easy to handle, studies using C. elegans have been conducted in space. Worms survive and grow well in space, and many functions of the worms 
59

60

61

62

63

64

65

66

67

are normal ((Higashitani et al. 2005; Szewczyk et al. 2008; Zhao et al. 2006). Muscle gene expression is slightly altered in space (Higashibata et al. 2006), as well as markers for aging (Honda et al. 2012). Hypergravity experiments on the ground show that behaviors and muscle structure of the worms are normal at a 100G force, although the FOXO transcription factor DAF16 translocates to the nucleus (Kim et al. 2007). However, no thorough studies on the effects of altering gravity to the motor neurons have been conducted.

Unlike vertebrate motor neurons that can exhibit plasticity (Inglis et al. 2000), C. elegans motor neurons are stable and fixed during adulthood (Sulston 1976; White et al. 1976). Motor neurons innervate 4 blocks of body wall muscle that line the ventral, dorsal, and lateral sides of the worm body to control forward and backward movement (Sulston \& Horvitz 1977). Since most of the changes in the motor neurons occur during development, the motor system in developing C. elegans is a better model for changes that occur in the adult vertebrate motor neurons than adult C. elegans. Particularly, D-type GABAergic motor neurons that are found on the ventral side of the animal extend circumferential axonal processes called commissures to the dorsal side where they join with the dorsal nerve cord to control movement (White et al. 1976). Six D-type motor neurons called the DD neurons are born embryonically and extend commisssures dorsally before the worm hatches from the egg shell. Another 13 D-type neurons called the VDs are born late in the first larval stage and then extend their commissures dorsally (White et al. 1976). Axon growth cones from the developing DD/VD motor neurons sense secreted attractive or repulsive cues along the body wall to find their targets in the dorsal side of the worm (Colavita et al. 1998; Hedgecock et al. 1990; MacNeil et al. 2009). from multiple cell types, and can be easily altered by genetic manipulation (Wadsworth 2002). 
82 We wondered what the effect of altering gravity would have on the development of the DD/VD

83 neurons. In this study we increased the gravity force on developing C. elegans worms by

84 centrifugation, resulting in a hypergravity environment. We found high gravity significantly

85 increases the number of animals that had disrupted DD/VD axon projections, and we

86 characterized the defects we found in detail here.

\section{MATERIALS AND METHODS}

88 Nematode culture and strains. Animals were grown and maintained at $20^{\circ} \mathrm{C}$ on Nematode

89 Growth Medium (NGM) plates seeded with E.coli OP50 as described previously (Brenner 1974).

90 Strains used for this study: N2, LG II: juIs 76 [(p)unc-25::GFP], LG IV: evIs82a [(p)unc-

91 129::GFP)] and LG X: $z d I s 5$ [(p)mec-4::GFP). The genetic background of the juIs 76 strain was

92 maintained by backcrossing this strain with the N2 wild-type strain, which was effective in

93 maintaining a consistent axonal defect rate (see Quanitification of defects section below).

94 Nematode hypergravity cultivation tube: 1L NGM media was prepared similar to previous

95 protocols (Lewis 1995), except Difco granulated agar was substituted for Bactoagar (Lee et al.

96 2016). $1 \mathrm{ml}$ of NGM was placed into each $1.5 \mathrm{ml}$ centrifuge tube and then transferred to a heat

97 block set at $42^{\circ} \mathrm{C}$ to prevent immediate solidification of agar. After distribution into tubes, the

98 tubes were spun at $100 \mathrm{G}$ for $2 \mathrm{~min}$ to solidify the agar and create a surface in which the force of

99 gravity is perpendicular to the flat agar surface in the tube.

100 To create a small lawn of E. coli bacteria, a single colony of OP50 strain bacteria was inoculated

101 in LB broth and incubated in a shaker at $37^{\circ} \mathrm{C}$ overnight, then concentrated by spinning down

102 and removing the supernatant. The pellet was resuspended and $2 \mu 1$ of bacteria was added to the

103 surface of each tube and allowed to dry at room temperature for at least 24 hours before usage. 
104 Preparation of eggs and hypergravity exposure: Eggs were harvested by bleaching gravid worms 105 according to a standard protocol (Steiernagle 1999), and then washed and collected in M9 buffer.

$1061 \mu 1$ of this solution was counted to obtain an approximate egg population density. For

107 hypergravity experiments, 100-150 eggs were placed into the cultivation tube, and centrifuged in 108 a temperature-controlled micro-centrifuge (Thermo Scientific, Sorvall Legend Micro17R

109 Centrifuge) at the particular G-force (rpm values for 6G, 10G, 100G and 500G are $300 \mathrm{rpm}, 400$

$110 \mathrm{rpm}, 1200 \mathrm{rpm}$ and $2800 \mathrm{rpm}$, respectively) at $20^{\circ} \mathrm{C}$ for various exposure times. A control tube

111 (1G) maintained in a $20^{\circ} \mathrm{C}$ incubator was performed with every experiment. Worms were

112 assessed by microscopy at the L4 larval/young adult stage which is 60 hours after egg harvesting.

113 If the hypergravity exposure time was shorter than 60 hours, the tubes were placed in a $20^{\circ} \mathrm{C}$

114 incubator after the hypergravity exposure until 60 hours was reached. For $3 \mathrm{hr}$ acute exposure 115 experiments, 3 day old adult animals were exposed to $100 \mathrm{G}$ hypergravity for $3 \mathrm{hrs}$. For $60 \mathrm{hr}$ 116 exposure adult animal experiments, individual 6-day old adult animals were picked directly into 117 the cultivation tube and exposed to $100 \mathrm{G}$ hypergravity for 60 hours.

118 Microscopic analysis: After 60 hours, animals were washed with M9 buffer and collected into $1191.5 \mathrm{ml}$ tubes and allowed to settle on the bottom of the tube. After removing the supernatant, 120 animals were mounted onto glass slides with a dry $2 \%$ agarose pad. To immobilize the worms, 2 $121 \mu \mathrm{l}$ of $1 \mathrm{M}$ sodium azide was added onto the agarose pad. An epi-fluorescent microscope 122 (Olympus BX50) was used to visualize the GFP-labeled neurons. Imaging software (Nikon 123 Elements) was used for extended depth of field (EDF) images, as well as all others images.

124 Quantification of defects: Defects were scored by researcher microscopic observations. Animals 125 were scored as defective if one or more of the 19 DD/VD neurons showed any axonal defect 126 (branch, turn and extend, stop, join and reach). Occasionally, an abnormally high axonal defect 
127 rate $(50 \%$ or more) was observed even in normal $1 \mathrm{G}$ conditions. We censured and discarded all

128 the data from these days. We found that this abnormality may be due to random genetic

129 abnormalities within this strain's genome. Thus, we maintained the genetic background of the

$130 j u I s 76$ strain by backcrossing this strain with the N2 wild-type strain. Dorsal-ventral locations of

131 defects were approximated by eye and scored as: Ventral - $0 \%$ to $15 \%$ dorsal-ventral distance,

132 Ventral sublateral $-15 \%$ to $50 \%$ dorsal-ventral distance, Dorsal sublateral - $50 \%$ to $85 \%$ dorsal-

133 ventral distance, Dorsal $-85 \%$ to $100 \%$ dorsal-ventral distance. Student's T-test was performed

134 (Microsoft Excel) to analyse the significance of data. For statistical analysis with multiple sets of

135 data, single-factor ANOVA was performed (Microsoft Excel) to determine variance, and post-

136 hoc Bonferonni correction was performed to determine significance.

\section{RESULTS}

\section{Hypergravity induces DD/VD motor neuron axonal defects}

To study the effect of hypergravity on C. elegans biology, we used a tabletop refrigerated centrifuge to create a gravity force and designed a small worm cultivation tube from a $1.5 \mathrm{~cm}$ centrifuge tube filled with NGM agar and seeded with OP50 E. coli bacteria on top (Fig 1A). To visualize the DD/VD motor neurons, we used a C. elegans transgenic strain that expresses GFP under the control of the $u n c-25$ gene promoter. The $u n c-25$ promoter directs expression of glutamic acid decarboxylase, an enzyme necessary for the production of GABA neurotransmitter, in the DD/VD motor neurons as well as several other neurons (Jin et al. 1999;

147 McIntire et al. 1993). Using the (p)unc-25::GFP strain, a total of 19 commissural DD/VD axons 148 can be observed running circumferentially from the ventral to the dorsal side (Fig 1B). Eggs 
149 containing developing embryos were placed in the cultivation tube, and either spun in the

150 centrifuge to induce a high gravity force of $100 \mathrm{G}$ or placed in a $20^{\circ} \mathrm{C}$ incubator as a $1 \mathrm{G}$ gravity

151 control. There were no obvious differences observed by eye between $1 \mathrm{G}$ and $100 \mathrm{G}$ : the pace of

152 development in $1 \mathrm{G}$ and $100 \mathrm{G}$ was identical, and hermaphrodite worms at 100G seemed to

153 develop normally to gravid adult mothers with no noticeable defects in movement. This is

154 consistent with a previous study that cultivated $C$. elegans in a microfluidic compact-disc

155 cultivation system at $100 \mathrm{G}$ and showed that worms had normal growth and adult movement and

156 behaviors (Kim et al. 2007; Kim 2007)

157 After 60 hours, the worms had reached mid-late L4 larval stage. At 1G, most of the

158 animals showed normal DD/VD commissural projections that reached the dorsal nerve cord (1B

159 and C), although occasional defects could be observed (1I and J). However, in animals exposed

160 to $100 \mathrm{G}$ hypergravity for 60 hours, defective axonal projections could be seen more frequently.

161 When we quantified the defects, we found that $21 \% \pm 3.75$ (4 trials, 425 total animals) of worms

162 grown in $1 \mathrm{G}$ displayed axonal defects (Fig 1I). However, worms grown in 100G had at least one

163 axonal defect in over $60 \% \pm 3.29$ (4 trials, 447 total animals) of the worms, which is about a 3-

164 fold higher frequency than in $1 \mathrm{G}$ conditions (Fig 1I). Among the total axons we looked at, axonal

165 defects occurred at a frequency of $1.5 \% \pm 0.36$ (Fig $1 \mathrm{~J} ; 4$ trials, 8046 total axons). In $100 \mathrm{G}$,

166 defects occurred at a rate of $5.1 \% \pm 0.17$ (4 trials, 7650 total axons), an over 3 -fold increase

167 compared to $1 \mathrm{G}($ Fig $1 \mathrm{~J})$.

168 Next, we varied the hypergravity force to observe their effects on DD/VD axon

169 development. Forces of $10 \mathrm{G}$ to $500 \mathrm{G}$ increased the percent of worms with axonal defects by

170 approximately $30 \%$ over $1 \mathrm{G}$. Interestingly, the number of defects did not significantly differ

171 from $10 \mathrm{G}$ to $500 \mathrm{G}$. On the other hand, a force of $6 \mathrm{G}$, the minimum speed for our table top 
172 centrifuge, could not induce axonal defects (Fig 2A). Therefore it seems that a certain threshold

173 in gravitational force exists between $6 \mathrm{G}$ and $10 \mathrm{G}$ that induces axonal defects. For our

174 experiments, we decided to use a hypergravity force of $100 \mathrm{G}$, which is the force used in a

175 previous study (Kim et al. 2007).

\section{Hypergravity-induced axonal defects requires exposure during a specific period of}

\section{development}

We wondered if there was a critical period during development that hypergravity can

induce motor neuron axonal defects. Hence, we exposed embryos to hypergravity for different time periods from the embryo stage and assessed DD/VD commissural axon defects in these animals at the 60 hour point or 72 hour point. Exposure of developing C. elegans to $100 \mathrm{G}$ hypergravity for 18,60 , and 72 hours all resulted in an approximately 2.5 to 3 fold significant increase in axonal defects (Fig $2 \mathrm{~B}$ and $\mathrm{C}$; $\mathrm{n}$ trials $18 \mathrm{hr}=4,60 \mathrm{hr}=4,72 \mathrm{hr}=6$ ). A shorter exposure of 10 hours resulted only in a small increase in worms with axonal defects, and a 3 or 6 hour exposure did not increase axonal defects (Fig $2 \mathrm{~B}$ and $\mathrm{C}$; $\mathrm{n}$ trials $3 \mathrm{hr}=5,6 \mathrm{hr}=5,10 \mathrm{hr}=4$ ). We also exposed 3-day old adult $C$. elegans to $100 \mathrm{G}$ hypergravity for 3 hours, and 6-day old adult animals to $100 \mathrm{G}$ for 60 hours, but there were no significant increases in axonal defects in the adult animals ( $\mathrm{n}$ trials $3 \mathrm{hr}=5,60 \mathrm{hr}=5$ ). It is important to note that embryos recovered by bleaching the mothers are not at a synchronous stage of development. Instead, these embryos are within an approximately 6 hour range of development. This may explain why we observe an intermediate phenotype at 10 hours hypergravity exposure (Fig 2C). Taking this into consideration, with our methods a minimum of 18 hours in hypergravity is required for an

194 increase in axonal defects. 
What may be occurring in the DD/VD motor neurons during this time in development?

196 DD neurons are born during the embryonic stage on the ventral side of the animal and send

197 projections to the dorsal side well before hatching (Sulston 1976). The VD neurons, however are

198 not born until the late L1/early L2 stage, more than 20 hours after these eggs are harvested from

199 their mothers (Figure 2C). Thus, during this 18 hour critical exposure period, the VD neurons

200 likely have not yet been born. More surprising was when we restricted exposure to $100 \mathrm{G}$

201 hypergravity from 4 to 18 hours, we saw a decrease in induced axonal defects compared to the

202 full 18 hours to a point (Fig 2B, 2C; n trials=4), further illustrating the importance of early

203 exposure to hypergravity in the observed increase in axonal defects.

\section{Characterization of hypergravity-induced axonal defects}

We then characterized the DD/VD motor neuron axonal defects more carefully by counting the defects in each commissure. We first checked the structure of the DD neurons in the

L1 stage immediately after a 15 hour hypergravity exposure and found that $100 \%$ of DD neuronal commissures are normal at this time point (Table 1). This was puzzling, since this meant that the defects we see in $1 \mathrm{G}$ at 60 hours likely arise after the mid-L1 stage, either by some alteration of the DD axons, or some predisposed developmental change to the yet-born VD neurons. This led us to characterize each of the axonal defects in the DD/VD neurons induced by 60 hour 100G hypergravity exposure more thoroughly. 
217 Therefore, we considered DD1/VD2 as one commissure, and count a total of 18 DD/VD

218 commissures. At normal $1 \mathrm{G}$ gravity conditions, occasional axon defects were observed in most

219 commissures spanning the anterior and posterior ends of the worm (Fig 3B). We found that

220 certain commissures had a higher probably of having axon defects than others. For instance, the

2215 commissures in the midbody region that include DD3, VD6, VD7, DD4, and VD8 accounted

222 for $50.93 \%$ of the total axonal defects whereas the 5 commissures on the anterior or posterior end 223 including VD1, VD2/DD1, VD12, DD6, and VD13 accounted for only $8.87 \%$ of the defects

$224(\mathrm{n}=630$ animals, total defects $=214)$. We also found that axonal defects were skewed towards the

225 VD commissures and that DD neurons showed less defects. We observed that $1.9 \%$ of all the VD

226 commissures were defective, whereas only $0.8 \%$ of the DD commissures showed defects (Table

227 1). In conclusion, although axonal outgrowth and guidance is a faithful biological process that

228 allows neuronal projections to reach their targets, the development of the DD and particularly the

229 VD motor neuron commissures are slightly error prone.

230

While defects sporadically occur during the development of the DD and VD axons in 1G

231

232

233

234

235

236

237

238

239 gravity conditions, $100 \mathrm{G}$ hypergravity clearly aggravates this error rate. In $100 \mathrm{G}$, the rate of defective axons jumps from $0.98 \%$ to $2.75 \%$ for DD commissures and $2.16 \%$ to $6.21 \%$ for VD commissures (Table 1), a 2.81-fold and 2.88-fold increase, respectively. Although hypergravity induces more axonal defects, it does not significantly change the distribution of those errors. For every DD axonal defect there are about 2.2 VD defects at $1 \mathrm{G}$, and this ratio is remarkably maintained in $100 \mathrm{G}$. In respect to location along the worm body, the five midbody commissures still account for $58 \%$ of the axonal defects in $100 \mathrm{G}$, whereas the five anterior/posterior end neurons again only account for $8 \%$ of the errors $(n=631$ animals, total defects $=614)$. Hence, hypergravity only exacerbates the total axon defect rate. 

direction. We grouped the defects into four categories: Ventral, Ventral sub-lateral, Dorsal sub-

242 lateral, and Dorsal. At $1 \mathrm{G}$, we found that most of the defects $(68.7 \%)$ were located in the dorsal 243 sub-lateral region which is defined as the area dorsal of the lateral mid-line, but ventral to the 244 area of the dorsal nerve cord (Fig 3C, see Materials and Methods). Interestingly, this dorsal sub245 lateral bias in axonal defects was maintained or slightly higher at 100G (86.5\%). We observed a 246 decrease in the number of dorsal defects in 100G, however the overall dorsal side bias of errors $247(96.9 \%$ in $1 \mathrm{G}, 97.5 \%$ in $100 \mathrm{G})$ is quite consistent. Once again, we find that hypergravity does 248 not greatly alter the pattern of axonal defects observed in normal gravity conditions.

We also characterized the axon commissural defects based on morphology. We defined four categories of defects: branched, turn and extend, stop, and joined and reach (Fig 4A). Axons

251

252

253

254

255

256

257

258

259

260

261

that abruptly stop, branch, or turn and extend never reach the dorsal nerve cord, whereas axons that join together do reach the dorsal nerve cord. Most of the defect were in the turn and extend category at close to $80 \%$, while others were all less than $10 \%$. Interestingly, the distribution of axonal defects in $1 \mathrm{G}$ conditions was strikingly maintained in 100G hypergravity (Fig 4B). Taken together, we find that hypergravity increases the overall rate of DD/VD commissural axon defects compared to normal gravity, but does not alter the distribution of axon defects.

\section{Hypergravity-induced axonal defects are specific for the DD/VD motor neurons.}

In a previous study, it was reported that 100G hypergravity did not affect the structure of the chemosensory neurons ASI and ADF, and touch sensory neurons AVM, ALM, PVM, and PLM, as well as the function of the AWA olfactory sensory neurons (Kim et al. 2007). We 
262 exposed a strain of $C$. elegans that expressed GFP in the touch sensory neurons to $100 \mathrm{G}$ and

263 confirmed that the structure of the touch sensory neurons were not affected by $100 \mathrm{G}$

264 hypergravity in our cultivation system (Table 1). To determine whether the structure of other

265 motor neurons could be affected, we exposed a strain of $C$. elegans that expressed GFP in the

266 DA/DB cholinergic motor neurons. The axonal commissures from the DA/DB motor neurons

267 project from the ventral to the dorsal side of the animal similar to the DD/VD motor neurons.

268 However, 100G hypergravity for 60 hours did not induce any defects in the DA/DB motor

269 neuron axons (Table 1). Thus, the hypergravity-induced axonal defects we have observed and

270 characterized may be specific for the DD/VD GABAergic motor neurons.

\section{DISCUSSION}

The physiology and cellular functions of organisms are adapted to the $1 \mathrm{G}$ gravity

274

275

276

277

278

279

280

281

282

283 conditions here on earth. This is clearly demonstrated by the biological effects that altered gravity in spaceflight has on vertebrates, particularly muscle atrophy (Vandenburgh et al. 1999).

However broader effects of altered gravity at the cellular level remain obscure. Here, we evaluated the effect that high gravity has on motor neuron development in the nematode $C$. elegans and characterized these changes. $10 \mathrm{G}$ to $500 \mathrm{G}$ hypergravity induces DD/VD motorneuron axon defects in 30\% more animals than 1G. Most of the axon defects were found in the dorsal sublateral region, and none of the defective axons reached their targets in the dorsal nerve cord. We found that an $18 \mathrm{hr}$ exposure from the embryonic stage to the first larval stage was sufficient to cause the axonal defects, whereas an acute 3 hour or longer 60 hour exposure in the adult animal did not induce defects. Although hypergravity increases the overall DD/VD 
284 motor neuron axon defects compared to $1 \mathrm{G}$, it does not alter the distribution or the characteristics

285 of these defects. Finally, these defects seem to be specific for the DD/VD motor neurons.

286 Though we saw the effects of hypergravity at a range of G forces, we used 100G for most

287 of our experiments. A previous study applied a 100G force using a compact disc-type cultivation

288 apparatus (Kim et al. 2007). This study showed that hypergravity induced the nuclear

289 localization of the conserved FOXO transcription factor DAF-16, and this was dependent on the

$290 \mathrm{ENaC} /$ degenerin sodium channel that functions in mechanosensation. Many features in the worm

291 were preserved in 100G, including muscle structure, olfactory behavior, feeding behavior, and

292 the structure of several neurons ((Kim et al. 2007); Table 1). However, the effect of

293 hypergravity on the structure of $C$. elegans motor neurons had not been assessed yet. Although

294 our hypergravity cultivation system was different than the compact-disc type apparatus

295 previously used (Kim 2007), we confirmed that growth progressed normally, movement was

296 normal, and the structure of touch sensory neurons was normal ((Kim et al. 2007); Table 1).

Although hypergravity can increase axonal defects in the VD motor neurons, we still observe a range of DD/VD axonal defects in a portion of the control animals at normal gravity.

This is consistent with observations from other groups that have studied DD/VD commissures using the juIs 76 strain that expresses (p)unc-25::GFP (Caceres Ide et al. 2012; Lee et al. 2015). In addition to the juIs 76 strain, we used the oxIs 12 strain that also expresses (p)unc-47::GFP in the DD/VD neurons (McIntire et al. 1997). Interestingly, we did not detect any DD/VD axonal defects at either $1 \mathrm{G}$ or $100 \mathrm{G}$ in this strain (see supporting dataset). However, a previous report cautioned that the oxIs 12 transgenic strain interfered with dosage compensation and affected expression of X-linked genes involved in axon guidance such as unc-6/netrin and lon-2/glypican genes, altering axon guidance phenotypes in different genetic backgrounds (Gysi et al. 2013). In 
307 addition, previous studies using electron microscopy have shown that the DD motor neurons can 308 develop in slightly different locations in individual animals (White et al. 1976), and the number 309 of axons innervating the dorsal nerve cord can also vary in individual animals (Hedgecock et al. 310 1990). These studies, along with the other studies using the juIs 76 strain, provide evidence that

311 the development of D-type motor neurons may show slight differences between individuals.

312 Thus, we have confidence that our observations using the (p)unc-25::GFP strain are valid, and 313 this lends credence to the fact that DD/VD axon development may normally be error prone.

Two major questions remain unanswered: what causes the errors in DD/VD axonal development, and how is hypergravity increasing these errors? Clues to answer the first question may lie in the distribution of the axonal defects. We find that commissures in the mid-body

317 region tend to show more defects than the commissures at the ends of the animal. One possibility that may cause this to arise is that the length of the commissures are slightly longer in the midbody area than the ends of the animal allowing for the possibility for more errors. Other differences in the mid-body compared to the ends of the animal are structures such as gonads, vulva, and muscle that could interfere with the guidance or migration of the axons. In fact, a previous study showed that axonal regeneration is less robust in the mid-body region likely due to interfering structures (Wu et al. 2007). We also find more defects in VD axons compared to DD axons. DD axons develop much earlier than the VD axons, and we show that at the L1 stage, the DD axons are completely normal (Table 1). However, by the L4 stage, defects have arisen in those same DD axons. DD axons migrate to the dorsal cord during the embryonic stage, but the animal continues to grow to an adult. It is not known how the DD axons maintain and grow with the developing body, and we wonder whether these errors can arise in the axon during this process. 

itself might be strong enough to break or damage the axons. However, an acute exposure and even a long exposure to hypergravity in adult animals was not sufficient to cause damage to the axons (Fig 2B). In addition, DD/VD motor neuron axons in C. elegans have the ability to repair and regenerate after damage (Hammarlund \& Jin 2014). In our experiments, we exposed the worms to $100 \mathrm{G}$ for 18 and 36 hours, and then allowed the worms to recover until 60 hours at $1 \mathrm{G}$.

This is enough time to regenerate any axons damaged by $100 \mathrm{G}$ force, yet we still observe hypergravity-induced axon defects. Thus, we are not certain whether high gravity induces axon damage.

We show an 18 hour exposure early in development and during the $1^{\text {st }}$ larval stage is sufficient to cause the axon defects (Fig 2B, 2C). The VD neurons have yet to be born when the critical period of hypergravity exposure occurs, yet we clearly observe defects in the VD neurons. Thus, we assume that hypergravity may be affecting another cell or tissue rather than the VD neurons themselves. Hypodermis and muscle is a major source for axon guidance cues such as UNC-6/netrin, UNC-129/TGF-beta, and LON-2/glypican (Blanchette et al. 2015; Wadsworth 2002), and we wonder whether hypergravity can somehow be altering these cues. Further experiments to determine whether problems in axon repair, axon guidance, or growth cone formation occur as a result of hypergravity should be conducted.

In this study we have addressed the role that high gravity conditions have on DD/VD motor neuron development. What effect does low gravity have on motor neuron development? Previous studies in rats showed that development of the dendrites of spiny motor neurons during spaceflight could be altered (Inglis et al. 2000). Similar studies to our hypergravity study can be conducted in microgravity in C. elegans at the genetic level. These studies could clarify whether 
353 low gravity effects on motor neuron development can also contribute to the muscle weakness and

354 atrophy observed in astronauts during long-term spaceflight and habitation.

\section{ACKNOWLEDGEMENTS}

357 Strains were provided by Jeong-Hoon Cho at Chosun University, and the Caenorhabditis Genetic

358 Center, which is funded by NIH Office of Research Infrastructure Programs.

\section{REFERENCES}

361

362

363

364

365

366

367

368

369

370

371

372

Blanchette CR, Perrat PN, Thackeray A, and Benard CY. 2015. Glypican Is a Modulator of Netrin-Mediated Axon Guidance. PLoS Biol 13:e1002183. 10.1371/journal.pbio.1002183

Brenner S. 1974. The genetics of Caenorhabditis elegans. Genetics 77:71-94.

Caceres Ide C, Valmas N, Hilliard MA, and Lu H. 2012. Laterally orienting C. elegans using geometry at microscale for high-throughput visual screens in neurodegeneration and neuronal development studies. PLoS One 7:e35037. 10.1371/journal.pone.0035037

Colavita A, Krishna S, Zheng H, Padgett RW, and Culotti JG. 1998. Pioneer axon guidance by UNC-129, a C. elegans TGF-beta. Science 281:706-709.

DeFelipe J, Arellano JI, Merchan-Perez A, Gonzalez-Albo MC, Walton K, and Llinas R. 2002. Spaceflight induces changes in the synaptic circuitry of the postnatal developing neocortex. Cereb Cortex 12:883-891.

Fire A, Xu S, Montgomery MK, Kostas SA, Driver SE, and Mello CC. 1998. Potent and specific genetic interference by double-stranded RNA in Caenorhabditis elegans. Nature 391:806811. $10.1038 / 35888$ 
375 Genchi GG, Ciofani G, Polini A, Liakos I, Iandolo D, Athanassiou A, Pisignano D, Mattoli V,

376

377

378

379

380

381

382

383

384

385

386

387

388

389

390

391

392

393

394

395

396

and Menciassi A. 2015. PC12 neuron-like cell response to electrospun poly( 3-

hydroxybutyrate) substrates. J Tissue Eng Regen Med 9:151-161. 10.1002/term.1623

Gysi S, Rhiner C, Flibotte S, Moerman DG, and Hengartner MO. 2013. A network of HSPG core proteins and HS modifying enzymes regulates netrin-dependent guidance of D-type motor neurons in Caenorhabditis elegans. PLoS One 8:e74908.

10.1371/journal.pone.0074908

Hammarlund M, and Jin Y. 2014. Axon regeneration in C. elegans. Curr Opin Neurobiol 27:199-207. 10.1016/j.conb.2014.04.001

Hedgecock EM, Culotti JG, and Hall DH. 1990. The unc-5, unc-6, and unc-40 genes guide circumferential migrations of pioneer axons and mesodermal cells on the epidermis in $\mathrm{C}$. elegans. Neuron 4:61-85.

Hengartner MO, and Horvitz HR. 1994. Programmed cell death in Caenorhabditis elegans. Curr Opin Genet Dev 4:581-586.

Higashibata A, Szewczyk NJ, Conley CA, Imamizo-Sato M, Higashitani A, and Ishioka N. 2006. Decreased expression of myogenic transcription factors and myosin heavy chains in Caenorhabditis elegans muscles developed during spaceflight. J Exp Biol 209:3209-3218. $10.1242 / \mathrm{jeb} .02365$

Higashitani A, Higashibata A, Sasagawa Y, Sugimoto T, Miyazawa Y, Szewcyk NJ, Viso M, Gasset G, Eche B, Fukui K, Shimazu T, Fujimoto N, Kuriyama K, and Ishioka N. 2005. Checkpoint and physiological apoptosis in germ cells proceeds normally in spaceflown Caenorhabditis elegans. Apoptosis 10:949-954. 10.1007/s10495-005-1323-3 
397 Honda Y, Higashibata A, Matsunaga Y, Yonezawa Y, Kawano T, Higashitani A, Kuriyama K, 398 Shimazu T, Tanaka M, Szewczyk NJ, Ishioka N, and Honda S. 2012. Genes down-

Inglis FM, Zuckerman KE, and Kalb RG. 2000. Experience-dependent development of spinal regulated in spaceflight are involved in the control of longevity in Caenorhabditis

Jin Y, Jorgensen E, Hartwieg E, and Horvitz HR. 1999. The Caenorhabditis elegans gene unc-25 encodes glutamic acid decarboxylase and is required for synaptic transmission but not synaptic development. J Neurosci 19:539-548.

Lee RC, Feinbaum RL, and Ambros V. 1993. The C. elegans heterochronic gene lin-4 encodes

Lee J, Bandyopadhyay J, Lee JI, Cho I, Park D, and Cho JH. 2015. A role for peroxidasin PXN-1 in aspects of C. elegans development. Mol Cells 38:51-57. 10.14348/molcells.2015.2202 small RNAs with antisense complementarity to lin-14. Cell 75:843-854.

Lee TY, Yoon KH, and Lee JI. 2016. NGT-3D: a simple nematode cultivation system to study Caenorhabditis elegans biology in 3D. Biol Open 5:529-534. 10.1242/bio.015743 
419 Lewis JAF, J.T. 1995. Basic Culture Methods. In: Epstein HFS, D.C., ed. Caenorhabditis

420 elegans: Modern Biological Analysis of an Organism. 1995: Academic Press, Inc., 4-27.

421 MacNeil LT, Hardy WR, Pawson T, Wrana JL, and Culotti JG. 2009. UNC-129 regulates the

422 balance between UNC-40 dependent and independent UNC-5 signaling pathways. Nat

423 Neurosci 12:150-155. 10.1038/nn.2256

424

McIntire SL, Jorgensen E, Kaplan J, and Horvitz HR. 1993. The GABAergic nervous system of 425 Caenorhabditis elegans. Nature 364:337-341. 10.1038/364337a0

426

427

428

429

430

431

432

433

434

435

436

437

438

439

440

McIntire SL, Reimer RJ, Schuske K, Edwards RH, and Jorgensen EM. 1997. Identification and characterization of the vesicular GABA transporter. Nature 389:870-876. 10.1038/39908

Steiernagle T. 1999. Maintenance of C. elegans. In: Hope I, ed. C elegans: A Practical Approach: Oxford University Press, 51-67.

Sulston JE. 1976. Post-embryonic development in the ventral cord of Caenorhabditis elegans. Philos Trans R Soc Lond B Biol Sci 275:287-297.

Sulston JE, and Horvitz HR. 1977. Post-embryonic cell lineages of the nematode, Caenorhabditis elegans. Dev Biol 56:110-156.

Szewczyk NJ, Tillman J, Conley CA, Granger L, Segalat L, Higashitani A, Honda S, Honda Y, Kagawa H, Adachi R, Higashibata A, Fujimoto N, Kuriyama K, Ishioka N, Fukui K, Baillie D, Rose A, Gasset G, Eche B, Chaput D, and Viso M. 2008. Description of International Caenorhabditis elegans Experiment first flight (ICE-FIRST). Adv Space Res 42:1072-1079. 10.1016/j.asr.2008.03.017

Vandenburgh H, Chromiak J, Shansky J, Del Tatto M, and Lemaire J. 1999. Space travel directly induces skeletal muscle atrophy. FASEB J 13:1031-1038. 
441 Wadsworth WG. 2002. Moving around in a worm: netrin UNC-6 and circumferential axon 442 guidance in C. elegans. Trends Neurosci 25:423-429.

443 White JG, Southgate E, Thomson JN, and Brenner S. 1976. The structure of the ventral nerve $444 \quad$ cord of Caenorhabditis elegans. Philos Trans R Soc Lond B Biol Sci 275:327-348.

445 Wu Z, Ghosh-Roy A, Yanik MF, Zhang JZ, Jin Y, and Chisholm AD. 2007. Caenorhabditis 446 elegans neuronal regeneration is influenced by life stage, ephrin signaling, and synaptic 447 branching. Proc Natl Acad Sci U S A 104:15132-15137. 10.1073/pnas.0707001104

448 Zhao Y, Lai K, Cheung I, Youds J, Tarailo M, Tarailo S, and Rose A. 2006. A mutational 449 analysis of Caenorhabditis elegans in space. Mutat Res 601:19-29.

450 10.1016/j.mrfmmm.2006.05.001

451 


\section{Figure 1 (on next page)}

100G hypergravity induces axonal defects in DD/VD motor neurons.

(A) Harvested embryos were exposed to hypergravity by centrifugation and then analyzed by microscopy as adults for neuronal defects. (B) (p)unc-25::GFP control animal at $1 \mathrm{G}$ gravity shows normal axonal commissures. Bar=100 $\mu$ (C) Magnified view of boxed area in (B). (D) (p)unc-25::GFP animal exposed to 100G hypergravity shows multiple axon commissural defects (white triangles) Bar=100 $\mu \mathrm{m}$. (E) Axonal defects in 100G exposed animals. Bar=50 $\mu m$. (F) 3D extended depth of field (EDF) image of (p)unc-25::GFP exposed to $100 \mathrm{G}$ hypergravity. Ventral in front, dorsal in back. Bar=100 um. (G) Magnified image of white box in (F) showing normal axonal commissures. Note the circumferential axons traveling along the body wall from the ventral to dorsal side during development. $(\mathrm{H})$ Magnified image of white box in (F) showing defective axon that turned and extended and formed branches before it approached the dorsal side of the animal. (I) Percent animals that display at least one axon defect for $1 \mathrm{G}$ and $100 \mathrm{G}$. Error bars represent SE. T-test was performed and ** indicates statistical significance $p<0.005$. (J) Percent axons that are defective in $1 G$ and 100 G. T-test was performed and $* * *$ indicates statistical significance $p<0.0005$. Error bars represent SE. 
PeerJ

A.

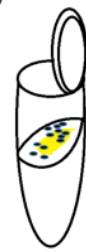

Centrifugation

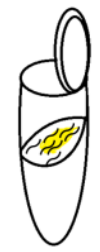

Manuscript to be reviewed

B.

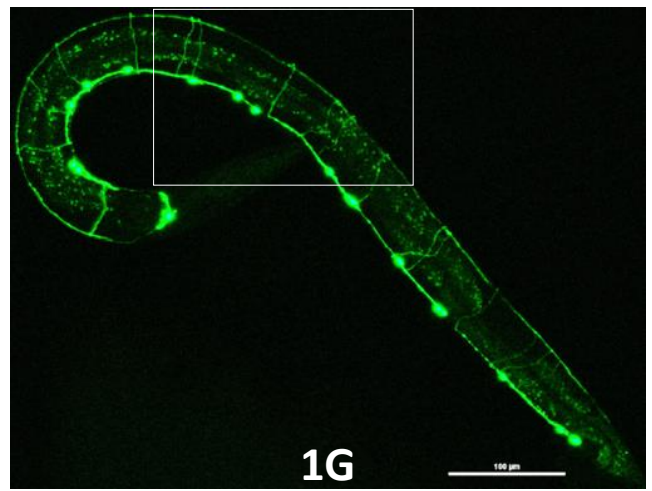

C.

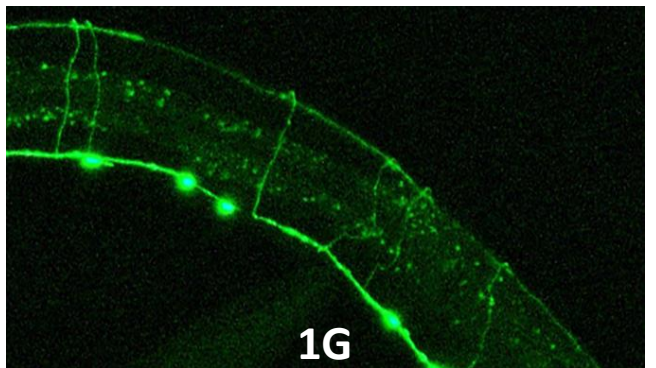

F.

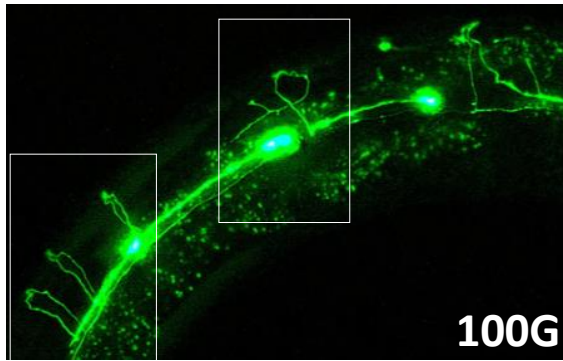

I
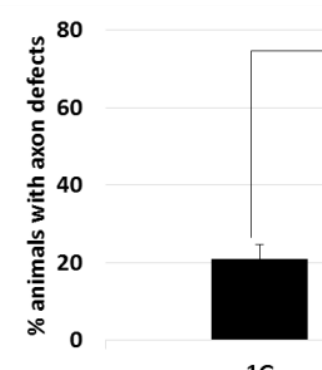

$* *$

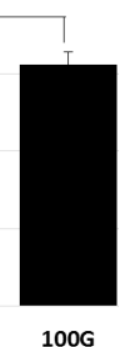

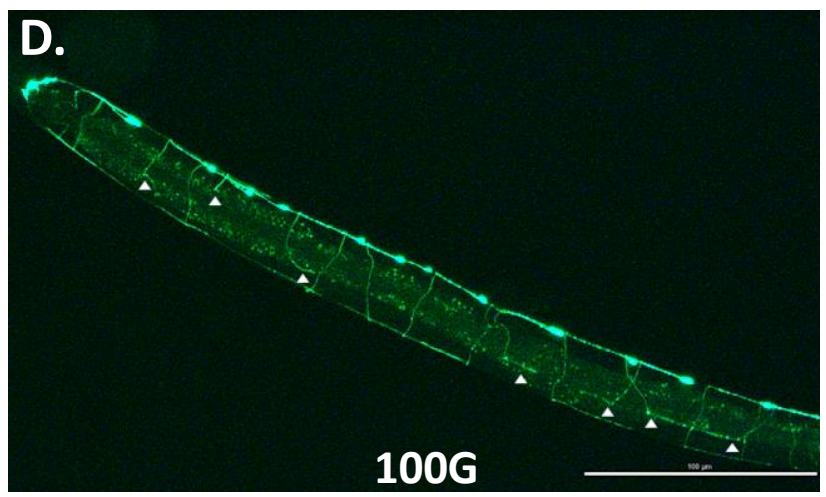

E.

$100 \mathrm{G}$

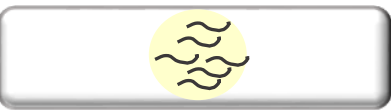

\section{Microscopic \\ Analysis}

$100 \mathrm{~g}$
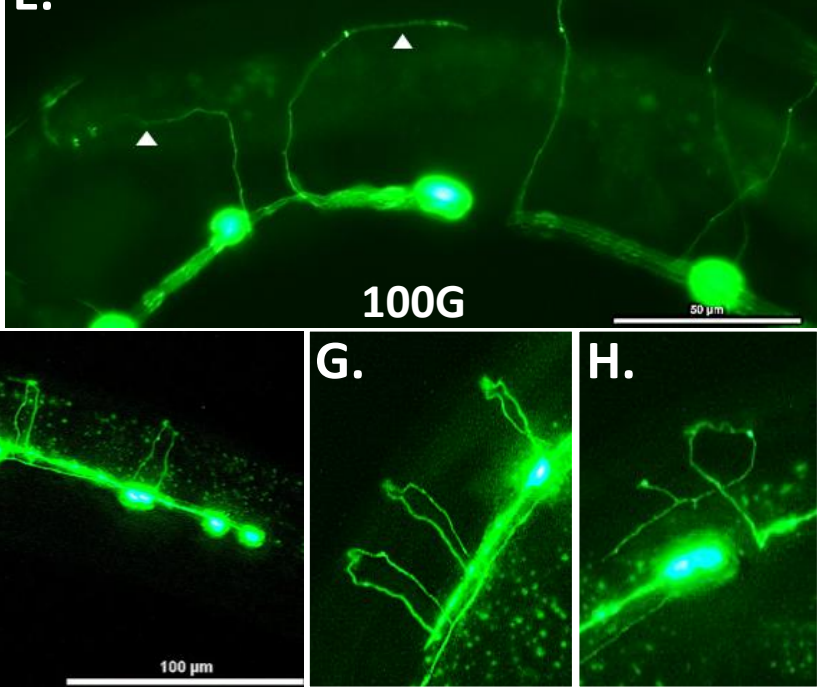

J. 6

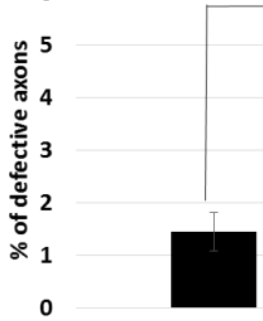

$1 \mathrm{G}$

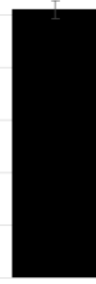

100G 


\section{Figure 2 (on next page)}

Hypergravity force and exposure time affect DD/VD neuron axon development.

(A) Animals were exposed to 1 to 500 G hypergravity for 60 hours after embryo harvesting. Gravity force of over $10 \mathrm{G}$ increased axonal defects. T-test was performed and ** indicates statistical significance $p<0.0005$. Error bars represent SE. (B) Animals were exposed to $100 \mathrm{G}$ hypergravity for various times after embryo harvesting, or to 3 or 60 hours during adulthood (far right bars). Grey bars=1G control, black bars=100G. Single-factor ANOVA and post-hoc Bonferonni correction was performed, $* *$ indicates statistical significance $p<0.005$. C) Data in (B) represented by exposure time over the major developmental events of $C$. elegans (top of graph). Green represents axonal defects induced, red represents axonal defects not induced, yellow represents axonal defects slightly induced. Bars represent SE. 
A. 80

PeerJ $t^{\circ} 70$

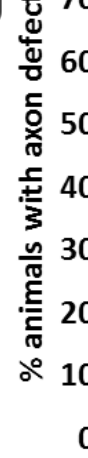

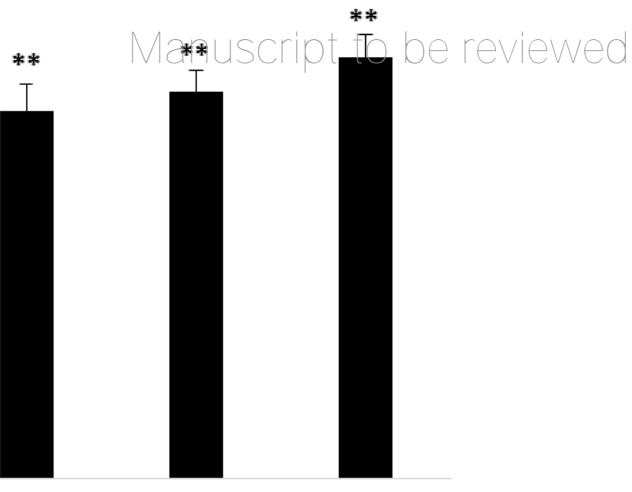

B.

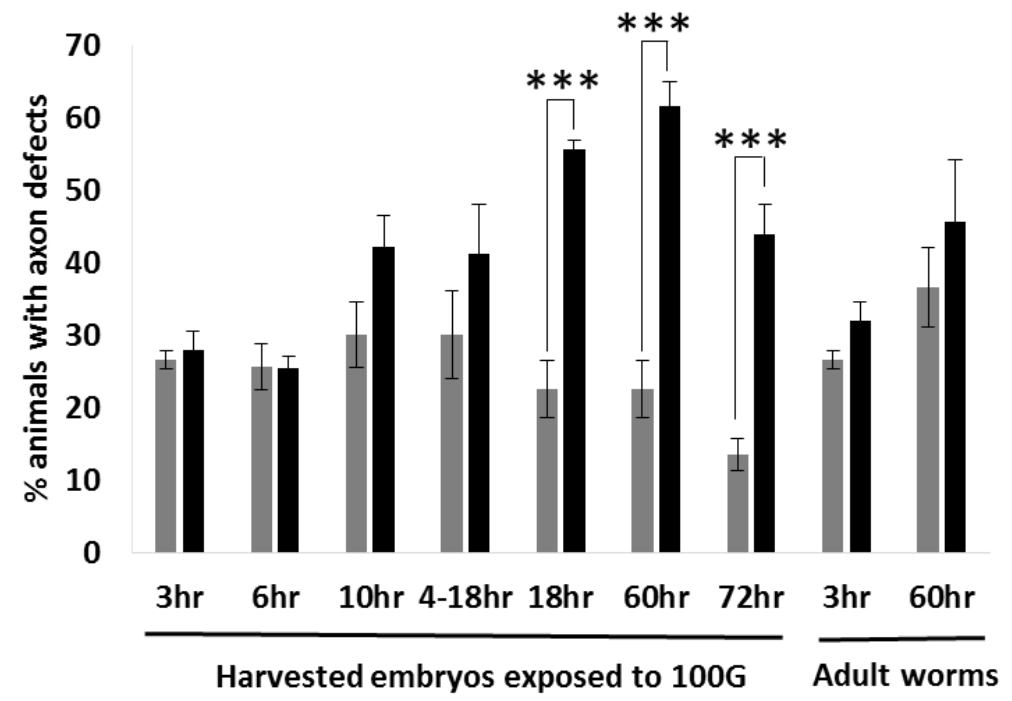

L1/L2

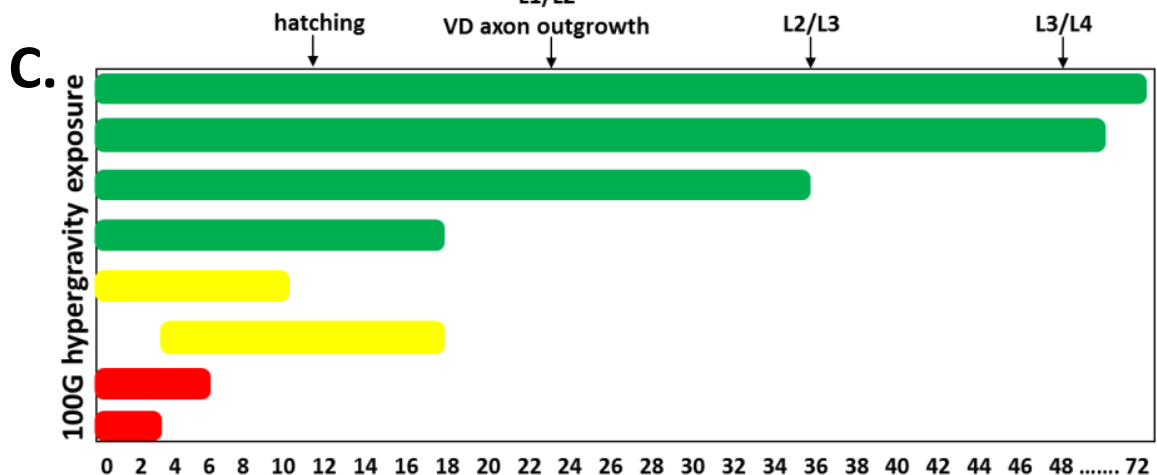

Hours after embryo harvesting 


\section{Figure 3 (on next page)}

Spatial distribution of axonal defects in $1 \mathrm{G}$ and $100 \mathrm{G}$ hypergravity exposed animals.

(A) Location of the DD and VD axon commissures along the anterior-posterior axis of the worm. VD2 and DD1 are overlapped commissures and are indistinguishable. (B) Axon defects for each VD/DD commissure. Top table shows the contribution of axon defects for each commissure to the total number of defects for $1 \mathrm{G}$ and $100 \mathrm{G}$. The percents for each commissure add up to $100 \%$ for $1 \mathrm{G}$ and $100 \mathrm{G}$, respectively. Heat maps in purple/lavender shades show the percents indicated in the legend on the right. $n=$ number of worms. Bottom table shows the percent defects for each individual commissure for $1 \mathrm{G}$ and $100 \mathrm{G}$. Heat maps in orange/yellow shades show the percents indicated in the legend on the right. (C) Localization of defects in the dorsal-ventral direction for $1 G$ animals. Defects were categorized into the four regions listed and percent of defects in each region is shown. (D) Localization of defects in the dorsal-ventral direction for 100G hypergravity exposed animals. 

Figure 4 (on next page)

Qualitative characterization of axonal defects in $1 \mathrm{G}$ and $100 \mathrm{G}$ hypergravity exposed animals.

(A) Axonal defects were categorized into the four groups shown here. (B) Quantification of axonal defects by category for $1 \mathrm{G}$ and $100 \mathrm{G}$ exposed animals. T-test was performed and none of the values were shown to be significantly different. Bars represent SE. 
$\ldots$

Branched

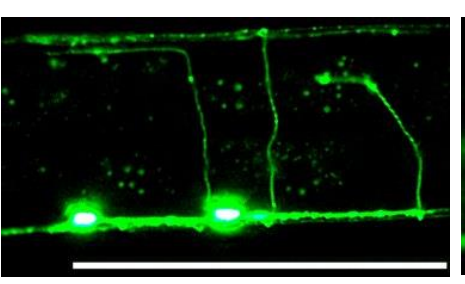

Turn and extend

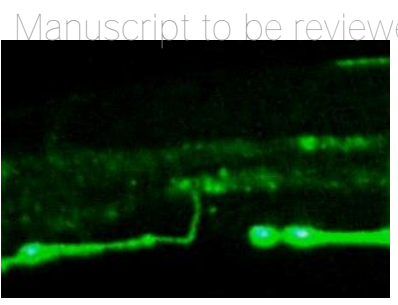

Stop

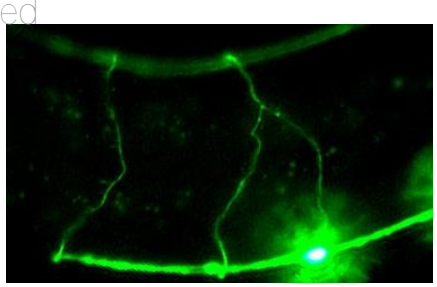

Join and reach

B.

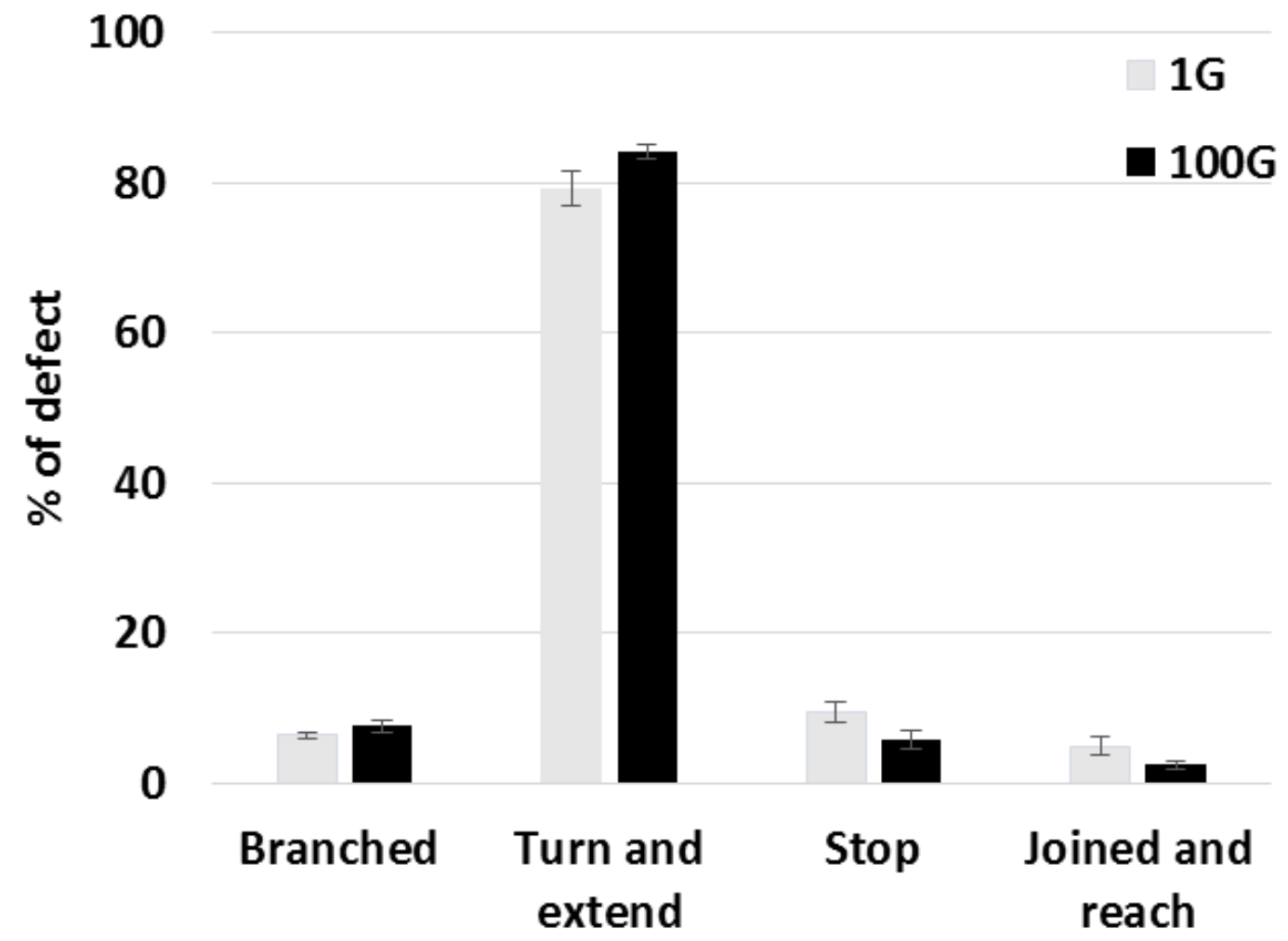




\section{Table 1 (on next page)}

Axon defects in different neuron types at 1G and 100G.

$\mathrm{n}=$ number of animals 
Table 1. Axon defects in different neuron types at 1G and 100G.

\begin{tabular}{|l|l|c|c|c|c|}
\hline \multicolumn{1}{|c|}{ Neuron type } & Reporter strain & $\begin{array}{c}\text { \% defective } \\
\text { axon }\end{array}$ & $\mathrm{n}$ & \% defective axon & $\mathrm{n}$ \\
\hline DD motor neurons & (p)unc-25::GFP & 0.98 & 630 & 2.16 & 631 \\
\hline VD motor neurons & (p)unc-25::GFP & 2.75 & 630 & 6.21 & 631 \\
\hline DD motor neurons (L1) & (p)unc-25::GFP & 0 & 375 & 0 & 316 \\
\hline Mechanosensory neurons & (p)mec-4::GFP & 0 & 50 & 0 & 45 \\
\hline Cholinergic neurons & (p)unc-129::GFP & 0 & 102 & 0 & 118 \\
\hline
\end{tabular}

$\mathrm{n}=$ number of animals 